\title{
RECURRENT DISLOCATION OF THE PATELLA AND OF THE SHOULDER
}

\author{
Their Association with Familial Joint Laxity
}

\author{
Cedric Carter, London, England
}

The Medical Research Council's Clinical Genetics Research Unit, Institute of Child Health, London

and

\author{
Rodney SweEtnam, London, England
}

Institute of Orthopaedics, Royal National Orthopaedic Hospital, London and Stanmore

Joint laxity is a feature of several dominantly inherited conditions. In Ehlers Danlos syndrome it is associated with hyperelasticity and fragility of the skin, in Marfan's syndrome with arachnodactyly, and in osteogenesis imperfecta with the well known bone changes. This laxity of ligaments may, however, occur alone. In any of these conditions the laxity of the joints may predispose to recurrent dislocation.

Joint laxity in more than one member of a family was first reported in the English literature by Finkelstein in 1916. The patient was a girl of two months with widespread generalised joint " hypermobility" and dislocation of both hips. Her mother, grandmother and two uncles also had joint laxity. A similar patient-a boy of seventeen months-was reported by Jahss in 1919, but the family history was less definite. The association of generalised joint laxity and recurrent dislocation of the shoulder has also been recorded (Sutro 1947), but there was no mention of any family inquiry.

In the most recent report on generalised joint laxity (Hass and Hass 1958) five patients were described, but none apparently had a relative similarly affected. Three of the patients had congenital dislocation of the hip, associated in one with dislocation of both radial heads and both inferior radio-ulnar joints. Of the remaining two patients, one had club feet and recurrent dislocation of the patella, and the other had dislocation of one sterno-clavicular joint.

In 1958 we drew attention to the association, in a single family, between familial joint laxity and recurrent dislocation of the patella (Carter and Sweetnam 1958). The pattern of inheritance of the joint laxity was that of a dominant gene.

That study led us to believe that joint laxity might be an important factor in recurrent dislocation of the patella and other joints, and that it might be found in a proportion of those patients with such dislocations. We now report a survey of patients with recurrent dislocation of the patella and with recurrent dislocation of the shoulder, designed to show how often these conditions are associated with, and are possibly caused by, familial joint laxity.

\section{FAMILIAL JOINT LAXITY AND RECURRENT DISLOCATION OF THE PATELLA}

We have traced the records of 111 patients suffering from recurrent dislocation of the patella who attended the Royal National Orthopaedic Hospital between 1948 and 1957. Letters were sent to each of them, asking whether any other member of their family had a similar condition.

Ninety-seven replies were received. Sixteen of the patients said that they had a relative similarly affected. All sixteen were examined, and a full family history was taken. In three instances two members of the same family replied, so that the total number of separate families was only thirteen. In three of these families we were unable to confirm the presence of recurrent dislocation affecting another relative, some other abnormality of the knee being discovered 
instead. Thus, ten families remained, of which the member, or members, who had attended the hospital for treatment constituted the index patients of this series. In two of these the underlying condition, both in the index patient and in the affected relative, was found to be familial joint laxity.

As a check on the reliability of the answers to the questionnaire, and to examine some non-familial cases for joint laxity, home visits were paid to twenty of those who had returned replies saying that they had no relatives affected. On detailed family inquiry the answer that no relative was affected was found to be right. Examination of these twenty patients showed that three had generalised joint laxity, and that in two of these the joint laxity, though not the dislocation, was present in other members of the family.

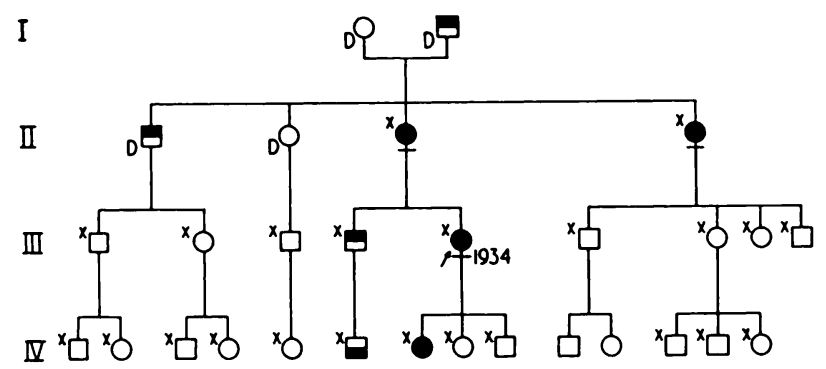

Fig. 1

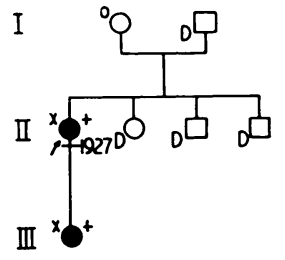

Fig. 2
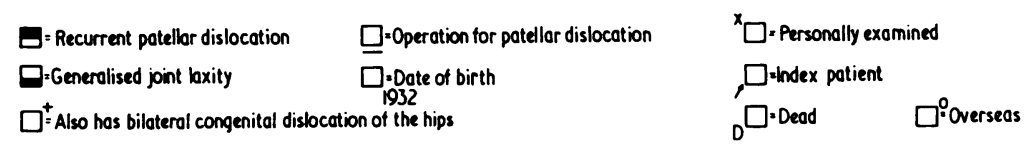

Figs. 1 AND 2

Pedigrees of the two families with recurrent dislocation of the patella associated with familial joint laxity.

Thus it appears that about 10 per cent of patients with recurrent dislocation of the patella have a relative similarly affected, and that familial joint laxity is not infrequently the cause of the recurrent dislocation when a relative is also affected. Familial joint laxity may also be the cause, though perhaps less often, of recurrent dislocation of the patella when there is no family history of dislocation.

In one other family with a pair of sisters who were both index patients the underlying condition was peroneal muscular atrophy (a condition known to be due to a specific dominant gene of varying manifestation).

In the remaining seven families, which contained more than one member with recurrent dislocation of the patella, but in which the index patient had no joint laxity, the affected relatives were as follows: son and daughter; aunt and mother; niece and uncle; mother; father; sister; niece.

The affected individuals in these seven families were carefully examined, clinically and radiologically. In some no abnormality could be discovered to which we could attribute the recurrent dislocation. In others only local abnormalities were found: for example, undue lateral mobility, underdevelopment of the patella, or hypoplasia of the lateral femoral condyle. No patient showed any significant knock-knee deformity. These local abnormalities were not always the same in the affected members of a single family, and their etiological significance is uncertain.

The two index patients and their families who had familial joint laxity are described below. 


\section{CASE REPORTS}

Case 1-A woman aged twenty-four (III 5 in the pedigree shown in Figure 1) first dislocated her left patella at the age of six. There were many recurrences and two years later a similar dislocation started in the right knee. She was treated unsuccessfully at the age of fourteen by plication of the quadriceps expansion on the medial side of both knees. When she was sixteen the left patella was excised, and great capsular laxity was found at operation. At the same age she suffered a traumatic dislocation of the right elbow. The right patella still dislocates.

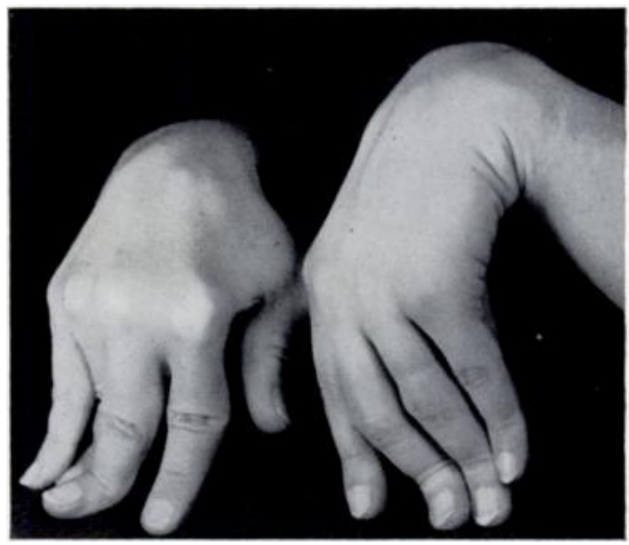

Fig. 3

Case 1-(III 5 in Figure 1). Laxity of the interphalangeal joints.

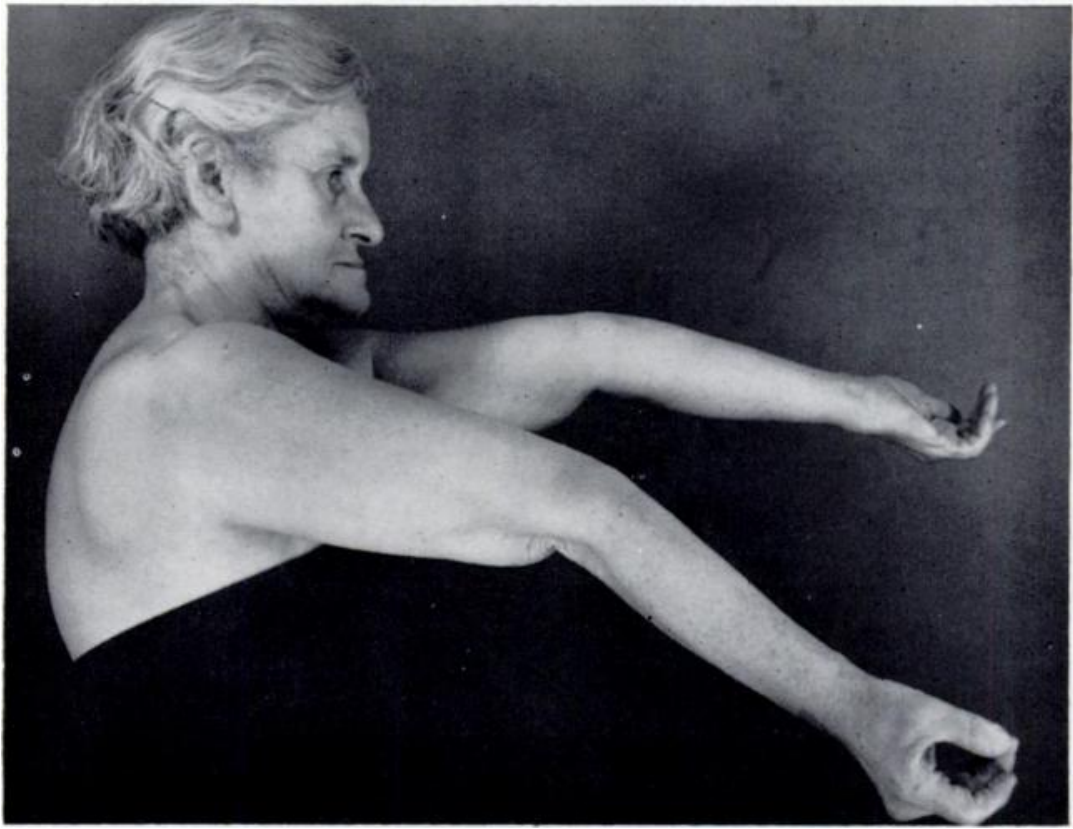

FIG. 4

Hyperextension of the elbows in the mother of the patient in Case 1 (II 3 in Figure 1).

On examination it was found that she had generalised joint laxity, the knees, elbows, wrists, and metacarpo-phalangeal joints all being involved (Fig. 3).

It is of interest that the patient's brother also had bilateral recurrent dislocation of the patellae, but had no generalised joint laxity. Such joint laxity was, however, present in his son. The laxity of the elbow joints in the mother (II 3 in Figure 1), a woman of fifty-five, is illustrated in Figure 4.

VOL. 42 B, NO. 4, NOVEMBER 1960 


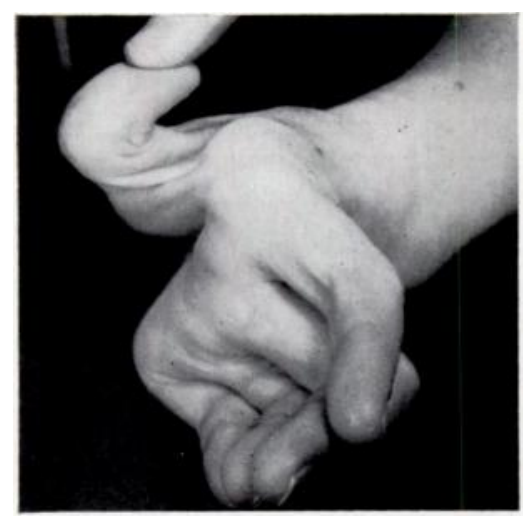

Fig. 5

Case 2-(II 1 in Figure 2). Joint laxity in the hand.
Case 2-A woman aged thirty-two (II 1 in the pedigree shown in Figure 2) was treated by $\mathrm{Mr} \mathrm{W}$. $\mathrm{H}$. Trethowan for bilateral congenital dislocation of the hips at the age of two. When seven years old she began to notice recurrent dislocation of both knee caps. She has since suffered traumatic dislocation of one shoulder, and has a permanent subluxation of both radial heads. This patient presented a remarkable degree of widespread joint laxity (Fig. 5).

Her only relative certainly affected was her daughter (a girl aged twelve, III 1 in Figure 2), who also had bilateral congenital dislocation of the hips and recurrent subluxation of both patellae. She, too, has generalised joint laxity of the same degree as her mother.

The index patient's sister and her two brothers were all dead. Her mother was alive and well in New Zealand and she has lost touch with her father. None of these relatives was considered by the patient to have had any joint dislocation or joint laxity.

FAMILIAL JOINT LAXITY AND RECURRENT DISLOCATION OF THE SHOULDER

A similar inquiry was made of patients attending the Royal National Orthopaedic Hospital, with recurrent dislocation of the shoulder, but these patients were also asked if they, or any of their relatives, were double-jointed. Answers were received from

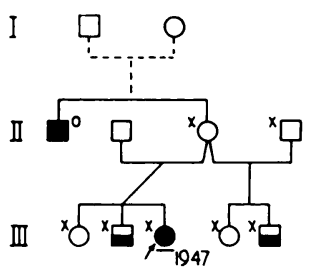

Fig. 6

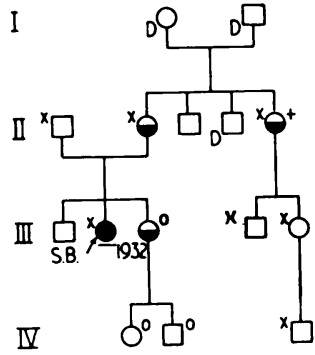

Fig. 7

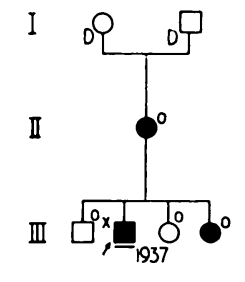

Fig. 8

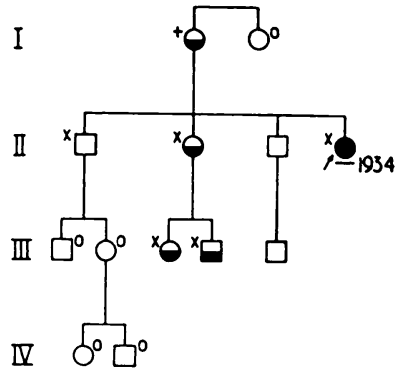

Fig. 9

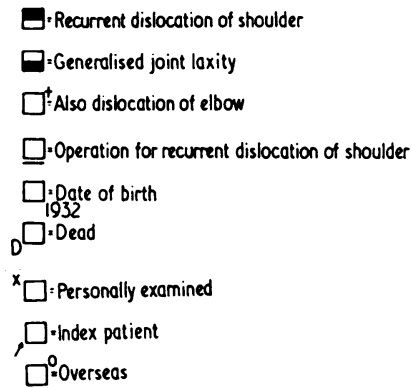

FIGS. 6 To 9

Pedigrees of the four families with recurrent dislocation of the shoulder associated with familial joint laxity.

forty patients, all of whom had attended for the first time between 1948 and 1957. Two of them reported that there was one relative similarly affected and also that other members of their respective families had double-joints. Another two reported that no relative was 
similarly affected but that they had some relatives who were double-jointed. As a check on the reliability of the answers, home visits were paid to ten of those who stated that they had no relative affected, and the answers were confirmed. On examination of these ten patients, and ten other patients with no family history, no generalised joint laxity was found. The case histories of the two patients with relatives similarly affected (Cases 3 and 5), and of the two patients with relatives who were double-jointed, but had no dislocations, are described below.

\section{CASE REPORTS AND PEDIGREES OF INDEX PATIENTS}

Case 3-A girl aged twelve (III 3 in the pedigree shown in Figure 6) dislocated her right shoulder when she was two years old. This recurred at about monthly intervals subsequently. Her left shoulder gave no trouble until eight years later, when it also dislocated. A successful Putti-Platt repair was performed on the right side in 1957.

Examination revealed generalised joint laxity. She had a somewhat lax skin and unusually short and broad feet. Unfortunately the mother and her brother were illegitimate, and so it was not possible to trace the family further back.

The patient's uncle aged thirty-one (II 1 in Figure 6), a seaman serving overseas, was not available for examination, but his medical records show that he has had treatment for recurrent dislocation of the right shoulder, and his younger sister gave a clear description of his generalised joint laxity.

Case 4-A woman aged twenty-seven (III 2 in the pedigree shown in Figure 7) knew from childhood that she and her sister were double-jointed. Her left shoulder first dislocated at the age of twenty, when she fell while skating. .Subsequent dislocations occurred frequently, often without noticeable injury, and she was always able to effect reduction by herself. A Bankart operation was performed in 1957, and she has had no further trouble. She presented all the features of

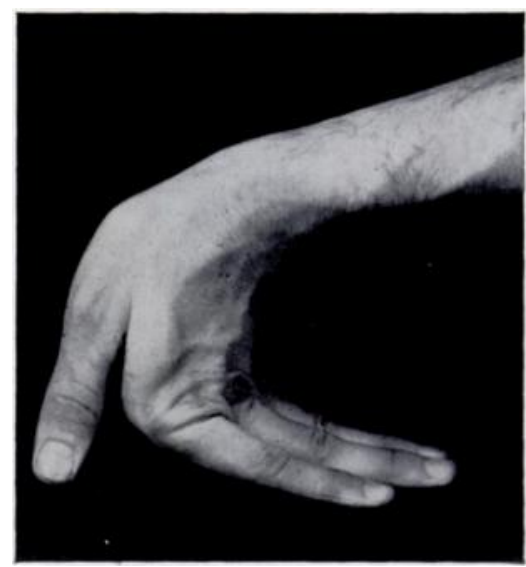

Fig. 10

Case 3 -(III 3 in Figure 6). Hyperextension of the metacarpo-phalangeal joints. familial joint laxity.

The sister of the patient was in America with her children and was not available for examination, but the patient and her mother gave a convincing description of the sister's joint laxity.

Case 5-A man aged twenty-two (III 2 in the pedigree shown in Figure 8), who was a South African undergraduate at Cambridge University, first suffered dislocation of his left shoulder when twenty years old. Frequent dislocation, usually without even minor injury, occurred thereafter, often several times a month. A Bankart operation was performed six months after the first dislocation, and he has had no recurrences.

On examination he was found to have generalised joint laxity (one hand is illustrated in Figure 10). The family were not available for examination, but the patient reported that his mother and one sister were double-jointed, and that both had recurrent dislocation of the shoulder.

We have accepted this intelligent man's description of his affected relatives as sufficiently reliable for us to include the family in the series.

Case 6-A woman aged twenty-five (II 4 in the pedigree shown in Figure 9) first dislocated her right shoulder when she was about twelve years old. Frequent further dislocation followed and often occurred without warning, particularly when she was reaching forward with her right arm. When she attended hospital it was found that on bringing the elbow forward the right humeral head could be felt to slip backwards out of the joint (Fig. 11). Surgical repair of the capsule was performed at the age of eighteen; at operation the joint was exposed from the back and the labrum found to be loose. It was reattached to the roughened glenoid and the redundant capsule overlapped. Fourteen months later posterior dislocation recurred and a bone block was inserted. One year later, however, the humerus again dislocated, but, this time, forwards. A third operation was performed, and on this occasion the front of the glenoid labrum was found detached and the capsule extremely lax. An anterior repair was performed, and there has been no recurrence.

On examination she was found to have generalised joint laxity. Examination of other members of her family showed that, while none of them had had any joint dislocation, several had generalised joint laxity.

VOL. 42 B, NO. 4, NOVEMBER 1960 


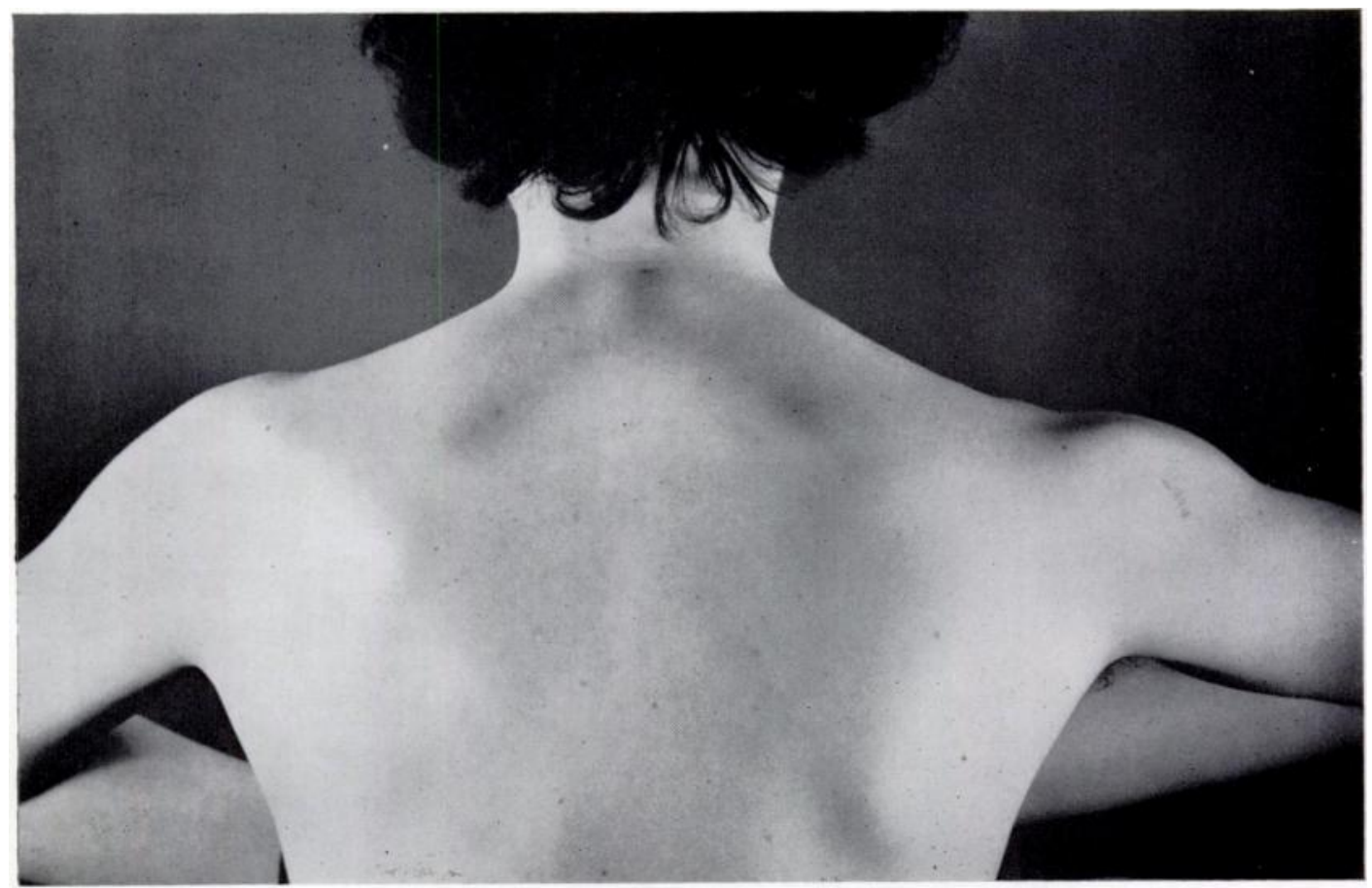

Fig. 11

Case 6-(II 4 in Figure 9). Posterior dislocation of the right shoulder.

\section{DISCUSSION}

Generalised joint laxity is not common. Two hundred orthopaedic out-patients were examined for the condition and only two were found to have generalised joint laxity of the degree found in the families reported in this paper. In one of the two it was a symptom of joint laxity, namely pain in the ankles from instability, which brought the patient to hospital.

It is clear from this investigation that joint laxity is a cause both of familial and sporadic cases of recurrent dislocation of the patella. There are, however, other ill-defined causes of the condition occurring in more than one member of a family. In contrast, familial joint laxity is rarely the cause of sporadic cases of recurrent dislocation of the shoulder, but, judging from our series, is the usual cause when the condition is familial.

The pattern of inheritance of generalised joint laxity in all these families is that given by a dominant gene or genes, as in those earlier reports in which the families were fully studied. There are some " skips" in the pedigrees in the sense that some of those who appear to have transmitted the gene are not themselves conspicuously loose-jointed; the reason may be that the diagnosis of joint laxity in mild degree is not easy. For example, the mother (II 3) of the index patient in Figure 6 with an affected brother has had affected children by two different husbands, but has no definite joint laxity herself. Again, the brother (III 4) of the index patient in Figure 1 has a son with generalised joint laxity, but he himself, though he has recurrent dislocation of both patellae, has only the laxity of the patellar ligaments that one associates with recurrent patellar dislocation.

There is no doubt that other joints may be affected in familial joint laxity. The mother and daughter shown in Figure 2 also had bilateral congenital dislocation of the hip, like the patients reported by Finkelstein (1916), Jahss (1919) and Hass and Hass (1958). The part played by familial joint laxity in cases of familial congenital dislocation of the hip is being further investigated by one of us. Congenital dislocation of the hip is not necessarily always present at birth, but the association of familial joint laxity with congenital club foot, reported 
by Key (1927), Hass and Hass (1958), Carter and Sweetnam (1958) is interesting; for, unless the association of club foot and joint laxity in these patients is coincidental, it indicates that some joints are already abnormally lax before birth.

Failure to obtain satisfactory surgical repair in patients with recurrent dislocation of the patella or shoulder cannot often be due to generalised joint laxity. Dislocation recurred after operation in only one of the index patients in this series. This patient (II 4 in Figure 9) suffered from recurrent posterior dislocation of the shoulder, and recurrence followed fourteen months after the first operation. A second repair was followed by anterior dislocation, and this required yet another repair before stability was finally achieved. A similar recurrence in a patient with generalised joint laxity has been observed before by Osmond-Clarke (1959).

\section{SUMMARY}

An inquiry was made of ninety-seven patients with recurrent dislocation of the patella and forty patients with recurrent dislocation of the shoulder to see how often they had a relative similarly affected, and also how often such dislocation is associated with, and perhaps caused by, familial joint laxity.

Ten of those with recurrent dislocation of the patella and two of those with recurrent dislocation of the shoulder were found to have a near relative with a similar dislocation. Familial joint laxity was found in two of the ten families with more than one member affected by recurrent patellar dislocation, and in both those with more than one member with recurrent dislocation of the shoulder. Familial joint laxity was also found in two out of twenty patients with recurrent dislocation of the patella who had no family history of similar dislocation; but in none out of twenty patients with recurrent dislocation of the shoulder and who had no family history of similar dislocation.

Familial joint laxity may be the only cause of recurrent dislocation of the shoulder occurring in more than one member of the family. But there are other, as yet undefined, causes of familial recurrent dislocation of the patella.

We wish to thank the surgeons of the Royal National Orthopaedic Hospital for permission to investigate the families of their patients. It is with pleasure we acknowledge the help of $\mathrm{Mr} \mathrm{H}$. Jackson Burrows and $\mathrm{Dr} \mathrm{J}$. A Fraser Roberts in the preparation of this paper. We should also like to thank the staffs of the Records Department and the Medical Photographic Department of the Institute of Orthopaedics, and the Department of Medical Illustration at the Hospital for Sick Children, for their help.

\section{REFERENCES}

Carter, C., and Sweetnam, R. (1958): Familial Joint Laxity and Recurrent Dislocation of the Patella. Journal of Bone and Joint Surgery, 40-B, 664.

Finkelstein, H. (1916): Joint Hypotonia. New York Medical Journal, 104, 942.

HASS, J., and HASS, R. (1958): Arthrochalasis Multiplex Congenita. Journal of Bone and Joint Surgery, 40-A, 663. JAHSs, S. A. (1919): Joint Hypotonia. New York Medical Journal, 109, 638.

KeY, J. A. (1927): Hypermobility of Joints as a Sex Linked Hereditary Characteristic. Journal of the American Medical Association, 88, 1,710.

Osmond-Clarke, H. O. (1959): Personal communication.

Sutro, C. J. (1947): Hypermobility of Bones due to "Overlengthened" Capsular and Ligamentous Tissues; a Cause for Intra-articular Effusions. Surgery, 21, 67. 